\title{
X-RAY EMISSION IN ABSENCE OF FLARES RELATED TO Ha ACTIVITY AND TYPE III BURST PRODUCTION
}

\author{
S. R. KANE \\ Space Sciences Laboratories, University of California, Berkeley, Calif. 94720, U.S.A. \\ R. W. KREPLIN \\ E. O. Hulburt Center for Space Research, Naval Research Laboratory, Washington, D.C. 20390, U.S.A. \\ and \\ M. J. MARTRES, M. PICK, and I. SORU-ESCAUT \\ Observatoire de Paris, 92 Meudon, France
}

\begin{abstract}
Solar Phys.). The relationship between $\mathrm{H} \alpha$ absorption features, type III radio bursts and soft $\mathrm{X}$-ray emission has been examined in order to determine the characteristics of the particle acceleration process operating when a $\mathrm{H} \alpha$-flare may or may not be detectable. The $\mathrm{H} \alpha$ observations were made by Meudon Observatory with a $\mathrm{H} \alpha$ telescope fitted with a $0.75 \AA$ band pass Lyot filter. During a $10 \mathrm{~s}$ period, three pictures were obtained - one at the $\mathrm{H} \alpha$ line center, one at $\mathrm{H} \alpha+0.75 \AA$ and one at $\mathrm{H} \alpha-0.75 \AA$. This sequence of three pictures was repeated every one minute. Each picture covered a rectangular area $18 \times 24 \mathrm{~mm}^{2}$, the diameter of the complete solar image being $38 \mathrm{~mm}$ on this scale. In addition, Meudon $\mathrm{H} \alpha$ films of the whole solar disc were also used. The X-ray observations were made with the University of California (Berkeley) experiment aboard the OGO-5 satellite and the NRL experiment aboard Solrad-9. The wavelength range covered was $0.5-20 \AA$. The type III radio data was obtained from two sources: The $169 \mathrm{MHz}$ radio-heliograph at Nancay which provided east-west position of the radio burst on the Sun with an accuracy of $\sim 1^{\prime}$ and the radio spectra measured by various ground based observatories. The findings are as follows:

Transient $H \alpha$ activity observed in the absence of reported flares is associated with production of type III radio and soft X-ray emission. Since such optical phenomena are much more frequent than flares themselves, we conclude that instabilities generating fast particles may be produced in the corona in a quasi-continuous way with coincident perturbations in the lower solar atmosphere.

The soft X-ray component is not necessarily the direct product of fast particles, but is probably associated with some type of heating since both the soft X-ray emission and the $\mathrm{H} \alpha$ features exhibit a comparable evolution. The type III bursts, when they are produced, occur near the maximum of this perturbation.

We identify the transient $\mathrm{H} \alpha$ activity (emission or absorption) with the existence of a metastable situation which may or may not lead to the triggering of a flare.
\end{abstract}




\section{DISCUSSION}

Newkirk: Aren't your results similar to the observations which led Hyder to formulate his impact infall model of some flares? He suggested that in these flares the X-ray emission emerged from the shock wave produced by the impact of prominence material into the chromosphere.

Pick: I believe that Hyder observed red shifts and we observe both blue and red shifts.

Martin: What is the spatial resolution of the $\mathrm{H} \alpha$ observations that you have used in your study.

Martres: Approximately 2".

Enome: What is the position of the absorbing feature in $\mathrm{H} \alpha$ in the active region? Is it located near the sunspot or the neutral line?

Pick: Along the inversion line of polarity. 